Federal Reserve Bank of Minneapolis

Research Department Staff Report 341

August 2004

\title{
Money and Capital as Competing Media of Exchange
}

\author{
Ricardo Lagos* \\ Federal Reserve Bank of Minneapolis \\ and New York University \\ Guillaume Rocheteau* \\ Federal Reserve Bank of Cleveland \\ and Australian National University
}

\begin{abstract}
We construct a model where capital competes with fiat money as a medium of exchange, and we establish conditions on fundamentals under which fiat money can be both valued and socially beneficial. When the socially efficient stock of capital is too low to provide the liquidity agents need, they overaccumulate productive assets to use as media of exchange. When this is the case, there exists a monetary equilibrium that dominates the nonmonetary one in terms of welfare. Under the Friedman Rule, fiat money provides just enough liquidity so that agents choose to accumulate the same capital stock a social planner would.
\end{abstract}

*We thank Randall Wright and seminar participants at the Australian National University, University of Paris 2, University of Sydney and the 2003 Meetings of the Society for Economic Dynamics. Lagos thanks the C.V. Starr Center for Applied Economics at NYU for financial support. Rocheteau has benefitted from the Summer Research Grant scheme of the School of Economics of the Australian National University. The views expressed herein are those of the authors and not necessarily those of the Federal Reserve Bank of Cleveland, the Federal Reserve Bank of Minneapolis or the Federal Reserve System. 


\section{Introduction}

We study an economy where real assets (capital goods) compete with fiat money as a medium of exchange, and we address the following questions: Can fiat money be valued - and useful to society - when real assets can be used as means of payment? Does the production of real assets provide enough liquidity to the economy? How do economies respond to liquidity shortages in the absence of fiat money?

We adopt the search-theoretic approach of Kiyotaki and Wright $(1989,1993)$ since it is wellsuited to study which objects endogenously emerge as media of exchange. In order to allow for competition between nominal and real assets, we follow Lagos and Wright (2000) and give agents periodic access to competitive markets where they can trade all assets. In addition, we let agents choose which assets to use as a means of payment in decentralized trades and impose no restrictions on their portfolios.

We establish a condition on fundamentals under which fiat money can be valued and socially beneficial. This condition states that money has a welfare-enhancing role when the capital stock that a social planner would accumulate is smaller than the stock of assets that agents need to conduct transactions. In the nonmonetary equilibrium, this liquidity shortage manifests itself as a premium on the rate of return on the assets that can be used as a medium of exchange, and this premium induces agents to overaccumulate capital. Capital plays two roles in this economy: it has a productive role, and it provides liquidity services. Its rate of return can be decomposed into a liquidity return, related to its role in the exchange process, and an intrinsic return associated with its productive role.

The introduction of fiat money helps to disentangle the productive use of the real asset from its liquidity use and induces agents to reduce the inefficiently high stock of capital goods. In the monetary equilibrium, money has the same rate of return as liquid capital since agents can exploit arbitrage opportunities in the centralized market. The Friedman Rule is the optimal monetary policy, and it induces the efficient accumulation of capital in the monetary equilibrium. Finally, there are also parameterizations under which the socially efficient capital stock provides enough liquidity to the economy, and in these circumstances fiat money is neither useful nor valued.

The notion of capital goods being used as media of exchange is not a pure theoretical 
abstraction. Einzig (1966, pp. 116-117) provides several accounts of primitive societies using capital goods - mainly cattle - as currency and presents evidence on the inefficiencies associated with such arrangements:

Goats and cattle were until comparatively recently the principal currency of a large part of Kenya. (...) In some districts livestock still constitutes the principal medium of exchange, in addition to serving social functions arising from the surviving tribal system. (...) Owing to the fact that cattle is, or was until recently, the sole currency of the Masai, they are grossly overstocked, far beyond requirements. (...) Before British control over the territories inhabited by them in Kenya and Tanzania became effective, the difficulties of over-stocking were overcome through raids on agricultural communities whose population was destroyed or enslaved, and whose cultivated land was turned into pasturage. When this could no longer be done, over-stocking tended to cause soil erosion. Once the cattle has eaten every blade of grass off the land, the soil turns into dust under the scorching, tropical sun, and the wind blows it away, leaving nothing but bare rocks. This problem of first-rate gravity preoccupied the Colonial Administrations in Kenya and other countries of East and South Africa. Deficiency of water supplies is also aggravated by overstocking. The remedy lies in inducing the Africans to abandon the monetary use of cattle and other livestock. (...) In the report of the Kenya Agricultural Commission Sir Daniel Hall suggested the issue of coins bearing the image of cows or goats, or to provide special tokens shaped like livestock and convertible into modern money, to bridge the psychological gap between the use of animal and mineral tokens of exchange.

Our model rationalizes Einzig's diagnosis - the use of a productive asset as a medium of exchange can result in an inefficiently large stock of the asset - and at the same time lends theoretical support to the Agricultural Commission's policy recommendation of introducing fiat money to mitigate this inefficiency.

There are also several interesting connections between our analysis and previous studies in monetary theory. The idea that commodity standards are undesirable because they distort the allocation of the commodity used in exchange can be traced back to Friedman (1960). The result that nonmonetary equilibria can exhibit overaccumulation of capital goods has been shown to be a feature of the overlapping generations (Wallace 1980) and Bewley models of 
monetary exchange (Bewley 1980). But while these models emphasize the store of value and insurance functions of money, our search-based model captures the role of money as a medium of exchange. Our work is also related to previous models of commodity money, such as Sargent and Wallace (1983), Kiyotaki and Wright (1989), Banerjee and Maskin (1996) and Burdett, Trejos and Wright (2001), which also find that commodity monies are socially inefficient arrangements. However, previous treatments of commodity money based on search theory differ from ours in that - as Banerjee and Maskin (1996, pp. 959-960) point out - they lack centralized markets and impose severe restrictions on agents' portfolios that limit the extent to which fiat money and real assets compete as media of exchange. ${ }^{1}$

The rest of the paper is organized as follows. Section 2 describes the model. Section 3 characterizes the optimal allocations. Section 4 formulates the individual decision problem and introduces the notion of equilibrium. Section 5 studies the coexistence of money and productive assets: it derives the conditions under which fiat money circulates and analyzes the implications for asset returns and welfare. Section 6 concludes. The appendix contains all proofs.

\section{The model}

The basic environment is that of Lagos and Wright (2000). Time is discrete and continues forever, and each period is divided into two subperiods where different activities take place. There is a $[0,1]$ continuum of agents and two types of perfectly divisible commodities - general and special goods. Each agent produces a subset and consumes a different subset of the special goods. Specialization is modeled as follows. Given two agents $i$ and $j$ drawn at random, there are three possible events. The probability that $i$ consumes something $j$ produces but not vice versa (a single coincidence) is denoted $\alpha$. Symmetrically, the probability that $j$ consumes something $i$ produces but not vice versa is also $\alpha$. In a single-coincidence meeting, we call buyer the agent who wishes to consume and seller the agent who produces. The probability neither wants anything the other can produce is $1-2 \alpha$, which means $\alpha \leq 1 / 2$. The specialization of agents over consumption and production of the special good give rise to a double coincidence

\footnotetext{
${ }^{1}$ In a recent paper, Aruoba and Wright (2004) also introduce capital in the Lagos-Wright model, but they assume it cannot be used as a medium of exchange. For a related analysis in a large-household framework, see Shi (1999).
} 
of wants problem. In contrast to special goods, general goods can be consumed and produced by all agents.

Special goods can only be produced during the first subperiod, while general commodities can only be produced during the second subperiod. In the first subperiod, agents participate in a decentralized market where each meeting is bilateral and is a random draw from the set of pairwise meetings. In this market the terms of trade are determined by bargaining. In the second subperiod agents produce general goods and can trade in a centralized market. All agents are anonymous, and there is no commitment or public memory, so all trade must be quid pro quo.

Let $u(q)$ and $-v(q)$ be the utility of consumption and production of special goods. Assume that $u(0)=v(0)=0, u^{\prime}(q)>0, v^{\prime}(q)>0, u^{\prime \prime}(q)<0, v^{\prime \prime}(q) \geq 0$, and that there exists $\bar{q}>0$ such that $u(\bar{q})=v(\bar{q})$. We also assume that $u^{\prime}(0)=\infty$ and $u^{\prime}(\infty)=0$. Consumption of $q$ units of the general good yields $q$ utils and production of $q$ units yields $-q$ utils. ${ }^{2}$ Individual consumption and production trajectories are sequences $\left\{q_{t}^{b}, q_{t}^{s}, c_{t}\right\}_{t=0}^{\infty}$, where $q_{t}^{b}$ is the quantity of search commodity consumed in period $t, q_{t}^{s}$ is the quantity of search commodity produced in period $t$, and $c_{t}$ is the net consumption of general goods in period $t$. An agent's lifetime utility is given by

$$
\mathbb{E}_{0} \sum_{t=0}^{\infty} \beta^{t}\left[u\left(q_{t}^{b}\right)-v\left(q_{t}^{s}\right)+c_{t}\right],
$$

where $\mathbb{E}_{0}$ is the expectation operator conditional on the information available at time 0 and $\beta \in(0,1)$ is the discount factor.

In addition to goods, there is an intrinsically useless, perfectly divisible and storable asset called money. Let $M_{t}$ denote the stock of money available in (the first subperiod of) period $t$. The gross growth rate of the money supply is constant and equal to $\gamma$; that is, $M_{t+1}=\gamma M_{t}$. Money is injected, or withdrawn if $\gamma<1$, through lump-sum transfers or taxes $T$. We use $\phi_{t}$ to denote the price of money in terms of general goods in the second subperiod of period $t$.

Lagos and Wright (2000) assume that both general and special goods are perishable, so money is the only asset that can be used as a medium of exchange. Here we also assume that

\footnotetext{
${ }^{2}$ For tractability, we only need that either the cost of production or the utility of consumption of the general good be linear. The quasi-linear specification eliminates wealth effects and helps keep the model tractable. Here we assume that both are linear because this simplifies the notation with no significant loss of generality. See Lagos and Wright (2000) and footnote 4 for details.
} 
special goods are perishable, but allow agents to accumulate general goods. Therefore, during the first subperiod, the feasible trades may involve exchanges of special goods, general goods, and money. In the second subperiod, trades may involve general goods and money. So in our setup, both money and general goods may serve as media of exchange. We give agents access to two different storage technologies for general goods. By storing $x_{i t}$ units of general goods at time $t$, an agent can get $i_{t+1}=F_{i}\left(x_{i t}\right)$ units of general goods before the search market of the following period. Alternatively, by storing $x_{k t}$ units of general goods at time $t$, an agent gets $k_{t+1}=F_{k}\left(x_{k t}\right)$ units of general goods after the search market of the following period. The key distinction is that the goods stored using the $F_{k}$ technology cannot be brought into the search market to be used as a medium of exchange. For simplicity we will refer to the general goods stored using technologies $F_{i}$ and $F_{k}$ as inventories and capital, respectively. ${ }^{3}$ Both $F_{i}$ and $F_{k}$ are strictly concave and satisfy $F_{i}^{\prime}(0)=F_{k}^{\prime}(0)=+\infty$ and $F_{i}(0)=F_{k}(0)=0$. Finally, we assume $\lim _{x_{i} \rightarrow \infty} F_{i}^{\prime}\left(x_{i}\right)<\beta^{-1}$ and $\lim _{x_{k} \rightarrow \infty} F_{k}^{\prime}\left(x_{k}\right)<\beta^{-1}$.

\section{The social optimum}

Consider the problem of a social planner wishing to maximize average expected utility. Let $q_{t}$ be the quantity traded in each match (since agents are anonymous, they are treated symmetrically by the planner) and $c_{t}$ the net consumption of general goods. The planner's problem is

$$
\begin{gathered}
\mathcal{W}=\max _{\left\{q_{t}, c_{t}, x_{i t}, x_{k t}\right\}_{t=0}^{\infty}} \sum_{t=0}^{\infty} \beta^{t}\left\{\alpha\left[u\left(q_{t}\right)-v\left(q_{t}\right)\right]+c_{t}\right\} \\
\text { s.t. } \quad c_{t}+x_{i t}+x_{k t} \leq i_{t}+k_{t} \\
i_{t+1}=F_{i}\left(x_{i t}\right) \\
k_{t+1}=F_{k}\left(x_{k t}\right)
\end{gathered}
$$

with $k_{0}$ and $i_{0}$ given. Equation (2) is a feasibility constraint for general goods: net consumption of general goods $\left(c_{t}\right)$ plus investment of general goods in the form of inventories and capital $\left(x_{k t}+x_{i t}\right)$ cannot exceed the available stock of general goods $\left(k_{t}+i_{t}\right)$. Equations (3) and (4)

\footnotetext{
${ }^{3}$ Aruoba and Wright (2004) is a special case of our setup with $F_{i}\left(x_{i}\right)=0$ for all $x_{i}$. Our model also nests the commodity money model of Burdett, Trejos and Wright (2001).
} 
are the laws of motion for capital and inventories. The first-order conditions are

$$
\begin{aligned}
\frac{u^{\prime}\left(q_{t}\right)}{v^{\prime}\left(q_{t}\right)} & =1 \\
\beta F_{k}^{\prime}\left(x_{k t}\right) & =1 \\
\beta F_{i}^{\prime}\left(x_{i t}\right) & =1
\end{aligned}
$$

for $t \geq 0$. Equation (5) defines $q^{*}$, the socially efficient quantity traded in each match. Equations (6) and (7) characterize the socially efficient levels of investment in capital and inventories. Let $x_{k}^{*}$ and $x_{i}^{*}$ denote the values of $x_{k t}$ and $x_{i t}$ that satisfy (6) and (7), and define $i^{*}=F_{i}\left(x_{i}^{*}\right)$ and $k^{*}=F_{k}\left(x_{k}^{*}\right){ }^{4}$ Having characterized the efficient allocation $\left(q^{*}, i^{*}, k^{*}\right)$, we turn our attention to the decentralized economy.

\section{Equilibrium}

For convenience, hereafter we suppress the time subscript $t$, and shorten $t+1$ to +1 and $t-1$ to -1 . Consider an agent who holds $m$ units of money, $k$ units of capital and $i$ units of inventories at the beginning of the first subperiod. We use $\mathbf{s}$ to denote his real portfolio $(z, i, k) \in \mathbb{R}_{+}^{3}$, where $z=\phi m$ is the real value of money holdings. Before entering the search market, each agent will choose the amount of money and inventories he brings into the search market to be used as means of payment. Let $\overline{\mathbf{s}}=(z, i, 0)$ denote the quantities of liquid assets owned by the agent, and let $\mathbf{a} \leq \overline{\mathbf{s}}$ denote the portfolio he actually chooses to carry into the search market. We will subsequently refer to a as the agent's "liquid portfolio."

The terms at which a pair of agents will trade in the search market depend on their liquid portfolios. When the buyer and seller hold liquid portfolios a and $\mathbf{a}^{\prime}$, the terms of trade are $\left[q\left(\mathbf{a}, \mathbf{a}^{\prime}\right), \mathbf{d}\left(\mathbf{a}, \mathbf{a}^{\prime}\right)\right]$, where $q\left(\mathbf{a}, \mathbf{a}^{\prime}\right) \in \mathbb{R}_{+}$is the quantity of special good traded and $\mathbf{d}\left(\mathbf{a}, \mathbf{a}^{\prime}\right)=$ $\left(d_{z}, d_{i}, d_{k}\right) \in \mathbb{R}_{+}^{3}$ represents the transfer of real assets (in terms of general goods) from the buyer to the seller. Since capital cannot be brought into the search market, $d_{k}=0$. The quantities of real money balances and inventories paid to the seller are $d_{z}$ and $d_{i}$.

\footnotetext{
${ }^{4}$ Notice that (6) and (7) imply that along the optimal path, $k_{t}=k^{*}$ and $i_{t}=i^{*}$ for all $t$. In other words, the capital stocks jump to their steady state values in one period. This is simply due to the fact that the planner's problem is linear in $c_{t}$. For example, if the return function was instead $\alpha\left[u\left(q_{t}\right)-c\left(q_{t}\right)\right]+U\left(c_{t}\right)$, with $U^{\prime}>0$ and $U^{\prime \prime} \leq 0$, we would have (focusing on interior solutions) the two standard Euler equations $\beta U^{\prime}\left(c_{t+1}\right) F_{k}^{\prime}\left(x_{k t}\right)=U^{\prime}\left(c_{t}\right)$ and $\beta U^{\prime}\left(c_{t+1}\right) F_{i}^{\prime}\left(x_{i t}\right)=U^{\prime}\left(c_{t}\right)$, instead of $(6)$ and $(7)$.
} 
Let $V(\mathbf{s})$ be the value function of an agent who owns portfolio $\mathbf{s}$ when he enters the search market, and $W(\mathbf{s})$ be the value function when he enters the centralized market. The value functions satisfy the following Bellman equation:

$$
\begin{aligned}
V(\mathbf{s})= & \max _{\mathbf{a} \leq \overline{\mathbf{s}}}\left\{\alpha \int\left\{u\left[q\left(\mathbf{a}, \mathbf{a}^{\prime}\right)\right]+W\left[\mathbf{s}-\mathbf{d}\left(\mathbf{a}, \mathbf{a}^{\prime}\right)\right]\right\} d \mathbf{H}\left(\mathbf{a}^{\prime}\right)\right. \\
& \left.+\alpha \int\left\{-v\left[q\left(\mathbf{a}^{\prime}, \mathbf{a}\right)\right]+W\left[\mathbf{s}+\mathbf{d}\left(\mathbf{a}^{\prime}, \mathbf{a}\right)\right]\right\} d \mathbf{H}\left(\mathbf{a}^{\prime}\right)+(1-2 \alpha) W(\mathbf{s})\right\},
\end{aligned}
$$

where $\mathbf{H}(\cdot)$ denotes the distribution of liquid portfolios. The first term is the expected payoff from a single coincidence meeting where the agent buys $q\left(\mathbf{a}, \mathbf{a}^{\prime}\right)$ and goes on to the centralized market with portfolio $\mathbf{s}-\mathbf{d}\left(\mathbf{a}, \mathbf{a}^{\prime}\right)$. The second term is the expected payoff from a single coincidence meeting where the agent sells $q\left(\mathbf{a}^{\prime}, \mathbf{a}\right)$ and enters the centralized market with portfolio $\mathbf{s}+\mathbf{d}\left(\mathbf{a}^{\prime}, \mathbf{a}\right)$. The last term is the expected payoff from going to the centralized market without having traded in the decentralized market.

Let $\mathbf{x}$ denote the vector of choice variables $\left(x_{z}, x_{i}, x_{k}\right)$ of an agent in the centralized market where $x_{z}$ is the choice of money balances expressed in terms of general goods. In what follows we will focus on steady state equilibria where real balances are constant over time, which implies $\phi / \phi_{+1}=\gamma$. In this context, $z_{+1}=F_{z}\left(x_{z}\right)=x_{z} / \gamma$, and we can define a time-invariant function $\mathbf{F}(\mathbf{x})=\left[F_{z}\left(x_{z}\right), F_{i}\left(x_{i}\right), F_{k}\left(x_{k}\right)\right]$, which maps the investments of real balances, capital and inventories into next period's portfolio. The agent's problem in the centralized market is

$$
\begin{gathered}
W(\mathbf{s})=\max _{c, \mathbf{x} \geq 0}\left[c+\beta V\left(\mathbf{s}_{+1}\right)\right] \\
\text { s.t. } \quad c+\mathbf{p x}=\mathbf{p s}+\phi T,
\end{gathered}
$$

where $\mathbf{s}_{+1}=\mathbf{F}(\mathbf{x})$ and $\mathbf{p}=(1,1,1)$ is the vector of prices of the different assets in terms of general goods. ${ }^{5}$ Equation (9) is the budget constraint: the agent finances his consumption of general goods and his investment in capital, inventories and money using the lump-sum transfer $T$ and his initial portfolio s, whose value in terms of general goods is ps. The government uses the lump-sum transfer to inject money into the economy, so $T=(\gamma-1) M$.

Substituting (9) into (8), we have

$$
W(\mathbf{s})=\mathbf{p s}+\phi T+\max _{\mathbf{x} \geq 0}\left[\beta V\left(\mathbf{s}_{+1}\right)-\mathbf{p x}\right] .
$$

\footnotetext{
${ }^{5}$ Since fiat money is intrinsically useless, there always exists an equilibrium where it has no value, i.e., where $\phi=0$ and therefore $x_{z} \in\{0\}$.
} 
Equation (10) has several implications. First, $W(\mathbf{s})$ could be rewritten as $W(\mathbf{p s})$ : when entering the centralized market, agents only care about the total real value of their portfolio. Second, the optimal choice of $\mathbf{x}$ is independent of the initial portfolio $\mathbf{s}$. Third, $W(\mathbf{s}+\mathbf{d})-W(\mathbf{s})=\mathbf{p d}$. This last observation allows us to write

$$
\begin{aligned}
V(\mathbf{s})= & \max _{\mathbf{a} \leq \overline{\mathbf{s}}}\left\{\alpha \int\left\{u\left[q\left(\mathbf{a}, \mathbf{a}^{\prime}\right)\right]-\mathbf{p d}\left(\mathbf{a}, \mathbf{a}^{\prime}\right)\right\} d \mathbf{H}\left(\mathbf{a}^{\prime}\right)\right. \\
& \left.+\alpha \int\left\{\mathbf{p d}\left(\mathbf{a}^{\prime}, \mathbf{a}\right)-v\left[q\left(\mathbf{a}^{\prime}, \mathbf{a}\right)\right]\right\} d \mathbf{H}\left(\mathbf{a}^{\prime}\right)+W(\mathbf{s})\right\} .
\end{aligned}
$$

Now consider a meeting in the first subperiod between a buyer with portfolio s, who chose to bring a into the decentralized market, and a seller with portfolio $\mathbf{s}^{\prime}$, who chose to bring $\mathbf{a}^{\prime}$. The terms of trade $(q, \mathbf{d})$ are determined by generalized Nash bargaining,

$$
\max _{q, \mathbf{d} \leq \mathbf{a}}[u(q)+W(\mathbf{s}-\mathbf{d})-W(\mathbf{s})]^{\theta}\left[-c(q)+W\left(\mathbf{s}^{\prime}+\mathbf{d}\right)-W\left(\mathbf{s}^{\prime}\right)\right]^{1-\theta},
$$

where $\theta \in(0,1]$ is the bargaining power of the buyer. The constraint $\mathbf{d} \leq \mathbf{a}$ indicates that the buyer cannot spend more than his liquid portfolio. Using (10) the problem reduces to

$$
\max _{q, \mathbf{d} \leq \mathbf{a}}[u(q)-\mathbf{p d}]^{\theta}[-c(q)+\mathbf{p d}]^{1-\theta} \text {. }
$$

From the first-order conditions of this problem, the value of real assets the buyer needs to transfer to the seller in exchange for quantity $q \in\left[0, q^{*}\right]$ of output is $g(q)$, where

$$
g(q)=\frac{(1-\theta) c^{\prime}(q) u(q)+\theta u^{\prime}(q) c(q)}{(1-\theta) c^{\prime}(q)+\theta u^{\prime}(q)} .
$$

If $\mathbf{p a} \geq g\left(q^{*}\right)$ the buyer gets $q=q^{*}$ in exchange for liquid assets with a real value of $\mathbf{p d}=g\left(q^{*}\right)$. Conversely, if pa $<g\left(q^{*}\right)$, then the buyer gets the $q$ that solves $g(q)=$ pa in exchange for all his liquid assets; i.e., $\mathbf{d}=\mathbf{a}$. So when $\mathbf{p a} \geq g\left(q^{*}\right)$, the buyer receives the socially efficient quantity $q^{*}$ in exchange for a part $\mathbf{d} \leq \mathbf{a}$ of his liquid portfolio, else the buyer hands over all his liquid assets in exchange for a quantity smaller than $q^{*}$. Note that the quantity $q$ traded in the match only depends on pa, the real value of the buyer's liquid assets. This means that the seller's portfolio and the composition of the liquid part of the buyer's portfolio are irrelevant for determining the terms of trade in the decentralized market. To make this explicit hereafter we use $a$ to denote the real value of an agent's liquid portfolio upon entering the decentralized market; that is, $a=$ pa. Now the bargaining solution can be written as

$$
q(a)= \begin{cases}q^{*} & \text { if } a \geq g\left(q^{*}\right) \\ g^{-1}(a) & \text { if } a<g\left(q^{*}\right)\end{cases}
$$


Using (12), (11) becomes

$$
V(\mathbf{s})=\max _{a \leq \mathbf{p} \overline{\mathbf{s}}}\left[\alpha \mathcal{S}_{b}(a)+\alpha \mathcal{S}_{s}+W(\mathbf{s})\right],
$$

where $\mathcal{S}_{b}(a)=u[q(a)]-g[q(a)]$ and $\mathcal{S}_{s}=\int\left\{\mathbf{p d}\left(\mathbf{a}^{\prime}, \mathbf{a}\right)-v\left[q\left(\mathbf{a}^{\prime}, \mathbf{a}\right)\right]\right\} d \mathbf{H}\left(\mathbf{a}^{\prime}\right)$ are the gains from trade of a buyer and a seller implied by the bargaining solution. According to (13) the agent chooses his liquid portfolio in order to maximize his expected surplus in matches where he acts as a buyer. If the constraint $a \leq \mathbf{p} \overline{\mathbf{s}}$ is slack, then $a=g(\tilde{q})$ where $\tilde{q}$ satisfies $u^{\prime}(\tilde{q})=g^{\prime}(\tilde{q})$. In what follows, we assume that $\mathcal{S}_{b}^{\prime \prime}(a)<0$ for all $a<g\left(q^{*}\right)$ to guarantee that the agent's problem is concave. ${ }^{6}$

Lemma 1 (a) The agent solves

$$
\max _{\mathbf{x}, a_{+1}}\left\{-\mathbf{p x}+\beta\left[\alpha \mathcal{S}_{b}\left(a_{+1}\right)+\mathbf{p F}(\mathbf{x})\right]\right\}
$$

subject to $\mathbf{x} \geq 0$ and $a_{+1} \leq F_{z}\left(x_{z}\right)+F_{i}\left(x_{i}\right)$, where $x_{z} \in \mathbb{R}_{+}$if the equilibrium is monetary and $x_{z} \in\{0\}$ otherwise.

(b) Let $R(a)=1+\alpha\left(\frac{u^{\prime}[q(a)]}{g^{\prime}[q(a)]}-1\right)$. The agent's problem has a unique solution $\left(\mathbf{x}, a_{+1}\right)$ that satisfies

$$
\begin{aligned}
\beta F_{k}^{\prime}\left(x_{k}\right) & =1 \\
\beta R\left(a_{+1}\right) F_{i}^{\prime}\left(x_{i}\right) & =1 \\
\beta R\left(a_{+1}\right) F_{z}^{\prime}\left(x_{z}\right) & \leq 1=\text { if } x_{z}>0 \\
R\left(a_{+1}\right) & \geq 1=\text { if } a_{+1}<F_{z}\left(x_{z}\right)+F_{i}\left(x_{i}\right) .
\end{aligned}
$$

The maximization problem in part (a) states that the agent chooses a vector of investments $\mathbf{x}$ and the value of the liquid portfolio $a_{+1}$ he will take to the search market in order to maximize the expected discounted values of search, $\mathcal{S}_{b}\left(a_{+1}\right)$, and of his next period portfolio, $\mathbf{p F}(\mathbf{x})$, net of the cost of acquiring the portfolio, $-\mathbf{p x}$.

The first-order conditions (15)-(18) are quite intuitive. Condition (15) equates the price of general goods, 1 , to the discounted utility value from saving an additional unit of the general good as capital. Since utility for general goods is linear, the discounted value of an additional unit of capital to the agent is just its discounted net rate of return $\beta F_{k}^{\prime}$. Similarly, (16) equates

\footnotetext{
${ }^{6}$ This assumption is a restriction on $u(\cdot), v(\cdot)$ and $\theta$. It is satisfied, for instance, if $\theta$ is close to 1.
} 
the marginal return to investing an additional unit of general goods as inventories to the price of general goods. The total return on inventories can be factored into two parts. First, there is the technological return $F_{i}^{\prime}$, since agents partly invest in inventories simply to reap the benefits of the storage technology. But inventories, in contrast to capital, also yield a liquidity return $R \geq 1$. This reflects the fact that inventories can play an additional role as a medium of exchange.

Condition (17) states that if real money balances are to be held, then at the margin their expected discounted rate of return $(\beta R / \gamma)$ must equal the marginal cost of acquiring real balances, which is 1 if money has value. Relative to capital, money stands on the opposite extreme: its value stems exclusively from its liquidity return $R$. Condition (18) says that if inventories and real balances yield a liquidity return, i.e., if $R>1$, then the agent will choose to bring all his liquid assets into the search market. Finally, note that (15) is identical to (6), so the choice of capital in the search equilibrium always coincides with the social planner's choice.

Definition 1 A stationary equilibrium is a vector of individual choices $\left(\mathbf{x}, a_{+1}\right)$, terms of trade $(q, \mathbf{d})$ and a sequence of prices $\left\{\phi_{t}\right\}$ such that $\left(\mathbf{x}, a_{+1}\right)$ solves $(14), q=q\left(a_{+1}\right), g(q)=\mathbf{p d}$ and $\phi_{t}=x_{z} / M_{t+1}$. An equilibrium is monetary if $\phi_{t}>0$ and nonmonetary otherwise.

\section{Fiat money, capital and welfare}

We begin by studying the economy when fiat money does not circulate.

Proposition 1 There exists a unique nonmonetary equilibrium. If $i^{*} \geq g(\tilde{q})$, then $k=k^{*}$, $i=i^{*}$, and $q=\tilde{q}$. If $i^{*}<g(\tilde{q})$, then $k=k^{*}, i>i^{*}$ and $q<\tilde{q}$.

The efficient stock of inventories, $i^{*}$, is large enough to allow agents to trade $\tilde{q}$, the level of output that maximizes the buyer's surplus in the decentralized market, when the primitives $\left(F_{i}, \beta, u, v, \theta\right)$ are such that $i^{*} \geq g(\tilde{q})$. For instance, this condition will be satisfied for economies with very productive storage technologies or where agents are very patient. In this case $i^{*}$ provides buyers with enough liquidity to maximize their gains from trade and as a result, $R=1$ : the marginal net liquidity return on inventories is zero. Note that the buyer's desired quantity traded in the search market, $\tilde{q}$, is less than $q^{*}$ whenever $\theta<1$. So the nonmonetary allocations coincide with the planner's choices if and only if $i^{*} \geq g(\tilde{q})$ and $\theta=1$. 
When $i^{*}<g(\tilde{q})$, the socially efficient stock of inventories is not large enough to allow agents to trade $\tilde{q}$. In this case, agents accumulate inventories in excess of $i^{*}$ to trade them in the decentralized market, and hence the stock of inventories is inefficiently high. In this equilibrium inventories pay a liquidity premium $R>1$ and output in the search market is inefficiently low. This overaccumulation result is due to the fact that inventories play two roles: they are held as an intrinsically productive asset as well as a medium of exchange. When $R>1$, the overall return on inventories exceeds its "technological" return. On the other hand, since capital cannot serve as a medium of exchange, $F_{k}^{\prime}=\beta^{-1}>F_{i}^{\prime}$. This result is reminiscent of the dynamic inefficiencies that arise in overlapping generations models, where overaccumulation of the productive asset results when it serves an additional role as a store of value. Similarly, in Bewley-type models, overaccumulation of the productive asset results when it serves an additional role as a means to self-insure against idiosyncratic shocks.

Next, we focus on equilibria where money is used as a medium of exchange.

Proposition 2 There exists a $\bar{\gamma}>\beta$ such that a unique monetary equilibrium exists iff $i^{*}<$ $g\left(q^{*}\right)$ and $\gamma \in[\beta, \bar{\gamma})$. In addition, $\partial i / \partial \gamma>0, \partial q / \partial \gamma<0, \partial \mathcal{W} / \partial \gamma<0, k=k^{*}, \lim _{\gamma \downarrow \beta} i=i^{*}$, and $\lim _{\gamma \downarrow \beta} q=\tilde{q}$. The allocation of the monetary equilibrium converges to the allocation of the nonmonetary equilibrium as $\gamma \uparrow \bar{\gamma}$.

From Propositions 1 and 2 we see that a monetary equilibrium exists if and only if the socially optimal stock of inventories $i^{*}$ does not provide enough liquidity, i.e., if $i^{*}<g(\tilde{q})$. This means that at $i^{*}$, in the barter equilibrium agents still want to accumulate additional inventories because of their role as a medium of exchange; recall that $q<\tilde{q}$, and hence inventories pay a liquidity premium $R>1$ when $i^{*}<g(\tilde{q})$. In this case, agents face the following trade-off: getting closer to $\tilde{q}$ requires accumulating inventories in excess of $i^{*}$, reducing the intrinsic return on inventories below its efficient level. There is also an equilibrium where money is valued. Real money balances allow agents to cut back on inventories and at the same time get closer to $\tilde{q}$. Consequently, the monetary equilibrium Pareto-dominates the nonmonetary equilibrium.

In a monetary equilibrium (17) holds with equality and the liquidity return is $R\left(a_{+1}\right)=\gamma / \beta$. Substituting this into (16) reveals that inventories and money are both held if and only if $F_{i}^{\prime}\left(x_{i}\right)=1 / \gamma$, namely, if the return on inventories, $F_{i}^{\prime}$, equals the return on real balances, $\gamma^{-1}$. In contrast, the gross rate of return of capital is $F_{k}^{\prime}\left(x_{k}\right)=\beta^{-1}$, which exceeds $\gamma^{-1}$, the return 
on inventories and money. In equilibrium capital cannot be used as a medium of exchange and hence it pays an illiquidity premium equal to $\beta^{-1}-\gamma^{-1}$. Just as in the barter economy, $x_{k}$ is given by (15) and is always at its efficient level.

From the above discussion we see that money can be held if and only if there is a premium for liquidity in a nonmonetary economy with inventories $i^{*}$, i.e., if and only if $R\left(i^{*}\right)>1$. But if this is the case, then (16) implies that the stock of inventories is necessarily above its efficient level. This makes clear that fiat money helps mitigate the overaccumulation inefficiency due to the shortage of liquid assets. It also reveals why any monetary equilibrium with $\gamma<\beta$ will be inefficient: $q$ will be too low, $R>1$, and $i$ too high. In fact $i=i^{*}$ if $\gamma=\beta$, but $q$ will be lower than $q^{*}$ unless $\theta=1$ in addition to $\gamma=\beta$.

We conclude by extending our main results to the case of a constant returns to scale storage technology analyzed by Wallace (1980) in an OLG model and by Burdett, Trejos and Wright (2001) in a search model with indivisible money. In our notation $F_{i}\left(x_{i}\right)=A x_{i}$, with $A \geq 0$. The economy analyzed by Lagos and Wright (2000) corresponds to the case with $A=0$ (general goods are nonstorable). The planner's problem has no solution if $\beta A>1$, and the solution is indeterminate if $\beta A=1$. If $\beta A<1$, the planner sets $x_{i}^{*}=0$. There is no equilibrium if $\beta A>1$ (agents would want to accumulate unbounded amounts of inventories). Let $q^{n}$ denote the quantity traded in the decentralized market and $x_{i}^{n}$ the investment in inventories in a nonmonetary equilibrium. For $\beta A=1$ we have $q^{n}=\tilde{q}$ and $x_{i}^{n}$ is indeterminate. More interestingly, if $\beta A<1$ we have $q^{n}<\tilde{q}$, where $q^{n}$ solves $\frac{u^{\prime}(q)}{g^{\prime}(q)}=1+\frac{1-\beta A}{\alpha \beta A}$, and $x_{i}^{n}=\frac{g\left(q^{n}\right)}{A}>$ $0=x_{i}^{*}$. In this nonmonetary economy there is no technological reason to save in inventories, yet agents do so to be able to consume in the decentralized market.

To characterize the monetary equilibrium, notice that with this linear storage technology (16) becomes $\gamma A \leq 1$, with equality if $x_{i}>0$. So a monetary equilibrium exists for all $\gamma \in\left(\beta, A^{-1}\right)$, and this interval is nonempty if $\beta A<1$, namely, the condition that implies that the nonmonetary equilibrium is inefficient. Let $q^{m}$ denote the quantity traded in the search market and $x_{i}^{m}$ the investment in inventories in a monetary equilibrium. Agents accumulate no inventories in a monetary equilibrium $\left(\gamma A<1\right.$ implies $\left.x_{i}^{m}=x_{i}^{*}=0\right)$ and carry real balances equal to $g\left(q^{m}\right)$, where $q^{m}$ solves $\frac{u^{\prime}(q)}{g^{\prime}(q)}=1+\frac{\gamma-\beta}{\alpha \beta}$. Finally, notice that as in Proposition 2 , in this case we have $q^{n}<q^{m} \leq q^{*}$ and $x_{i}^{*} \leq x_{i}^{m}<x_{i}^{n}$. 


\section{Conclusion}

We constructed a model where real assets compete with fiat money as a medium of exchange and derived a condition on the fundamentals of the economy under which fiat money can be both valued and socially beneficial. The presence of valued fiat money is welfare-improving when the stock of capital goods that agents would choose to accumulate in the absence of a liquidity motive is too low to provide the liquidity they need for exchange purposes. In this case capital pays a liquidity premium and, in a nonmonetary equilibrium, agents overaccumulate capital goods. Under these circumstances the nonmonetary equilibrium is inefficient and it always coexists with a monetary equilibrium in which both capital and money circulate. Valued money increases welfare because it allows agents to uncouple the role of capital as a medium of exchange from its role as an intrinsically productive asset. Under the optimal monetary policy (the Friedman Rule), fiat money provides just enough liquidity so that the incentives to invest in capital are purely technological and agents choose to accumulate the same capital stock a social planner would implement. 


\section{Appendix}

Proof of Lemma 1. (a) Substitute (13) into (10) to obtain

$$
W(\mathbf{s})=\mathbf{p s}+\phi T+\max _{\mathbf{x} \geq 0, a_{+1} \leq \mathbf{p} \overline{\mathbf{s}}_{+1}}\left\{\beta\left[\alpha \mathcal{S}_{b}\left(a_{+1}\right)+\alpha \mathcal{S}_{s}+W\left(\mathbf{s}_{+1}\right)\right]-\mathbf{p x}\right\}
$$

where $\mathbf{s}_{+1}=\mathbf{F}(\mathbf{x})$ and $\overline{\mathbf{s}}_{+1}=\left(F_{z}\left(x_{z}\right), F_{i}\left(x_{i}\right), 0\right)$. Using the linearity of $W$ and ignoring the constants, the agent's problem becomes

$$
\max _{\mathbf{x} \geq 0, a_{+1} \leq \mathbf{p} \overline{\mathbf{s}}_{+1}}\left\{\beta\left[\alpha \mathcal{S}_{b}\left(a_{+1}\right)+\mathbf{p s}_{+1}\right]-\mathbf{p x}\right\},
$$

as stated in the Lemma. (b) Let $\Delta\left(\mathbf{x}, a_{+1}\right)=-\mathbf{p x}+\beta\left[\alpha \mathcal{S}_{b}\left(a_{+1}\right)+\mathbf{p} \mathbf{F}(\mathbf{x})\right]$, and write the

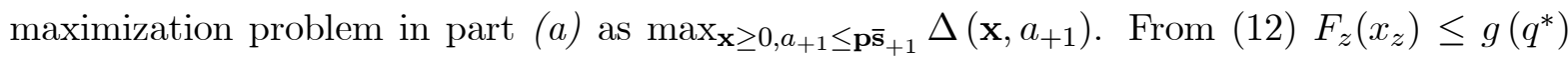
and $F_{i}\left(x_{i}\right) \leq \max \left[i^{*}, g\left(q^{*}\right)\right]$ so that the set for the control variables is compact. Since $\Delta\left(\mathbf{x}, a_{+1}\right)$ is continuous, a solution always exists, and it satisfies the first-order conditions (15)-(18). To establish uniqueness, consider two cases. First, if $i^{*} \geq g(\tilde{q})$, then $x_{z}=0, x_{i}=x_{i}^{*}, x_{k}=x_{k}^{*}$ and $a_{+1}=g(\tilde{q})$. Conversely, if $i^{*}<g(\tilde{q})$, then $a_{+1}=F_{z}\left(x_{z}\right)+F_{i}\left(x_{i}\right)$ and $R\left(a_{+1}\right) \geq 1$, which implies $a_{+1} \leq g(\tilde{q})$. Since $\mathcal{S}_{b}(a)$ is strictly concave for all $a \leq g(\tilde{q})$, it can be checked that $\Delta\left(\mathbf{x}, F_{z}\left(x_{z}\right)+F_{i}\left(x_{i}\right)\right)$ is strictly concave in $\mathbf{x}$ for all $F_{z}\left(x_{z}\right)+F_{i}\left(x_{i}\right) \leq g(\tilde{q})$. As a consequence, the first-order conditions (15)-(18) are necessary and sufficient for an optimum.

Proof of Proposition 1. Existence and uniqueness come from Lemma 1. From (15), $x_{k}=x_{k}^{*}$ so $k=k^{*}$. If $g(\tilde{q}) \leq i^{*}$, then the unconstrained solution $a_{+1}=g(\tilde{q}), x_{i}=x_{i}^{*}$ and $i=i^{*}$ is available. Conversely, if $g(\tilde{q})>i^{*}$, then $R\left(a_{+1}\right)>1$ (since $R\left(a_{+1}\right)=1$ would imply $g(\tilde{q}) \leq i^{*}$ ) and (16) yields $x_{i}>x_{i}^{*}$ and $i>i^{*}$. The fact that $q<\tilde{q}$ follows from $a_{+1}<g(\tilde{q})$.

Proof of Proposition 2. From (15)-(18) we see that in a monetary equilibrium $\left(x_{z}>0\right)$ the allocation $\left(\mathbf{x}, a_{+1}, q\right)$ is given by $F_{k}^{\prime}\left(x_{k}\right)=1, F_{i}^{\prime}\left(x_{i}\right)=1 / \gamma, R\left(a_{+1}\right)=\gamma / \beta, F_{z}\left(x_{z}\right)=a_{+1}-F_{i}\left(x_{i}\right)$ and $g(q)=a_{+1}$. From this, it follows that $k=k^{*}, \partial i / \partial \gamma>0$ and $\partial q / \partial \gamma<0$. For a monetary equilibrium, in addition we need $x_{z}>0$, i.e., $\Gamma(\gamma) \equiv a_{+1}(\gamma)-F_{i}\left[x_{i}(\gamma)\right]>0$. Since $\Gamma^{\prime}(\gamma)<0$ and $\lim _{\gamma \rightarrow \infty} \Gamma(\gamma)<0$, a monetary equilibrium exists iff $\Gamma(\beta)>0$, i.e., $g\left(q^{*}\right)-i^{*}>0$ and $\gamma<\bar{\gamma}$ defined by $\Gamma(\bar{\gamma})=0$. As $\gamma \rightarrow \bar{\gamma}, x_{z} \rightarrow 0$ and the allocation $\left(\mathbf{x}, a_{+1}, q\right)$ converges to the equilibrium allocation of the nonmonetary economy. As $\gamma \rightarrow \beta, R\left(a_{+1}\right) \rightarrow 1$, so $i \rightarrow i^{*}$ and $q \rightarrow \tilde{q}$. Finally we have $(1-\beta) \mathcal{W}=\alpha[u(q)-v(q)]$, so welfare is decreasing in $\gamma$. 


\section{References}

[1] Aruoba, S. Boragan, and Randall Wright (2004). "Search, Money and Capital: A Neoclassical Dichotomy," Journal of Money, Credit, and Banking (Forthcoming).

[2] Banerjee, Abhijit V., and Eric S. Maskin (1996). "A Walrasian Theory of Money and Barter," Quarterly Journal of Economics 111, 955-1005.

[3] Bewley, Truman (1980). "The optimum quantity of money," in Models of Monetary Economies, John Kareken and Neil Wallace, eds., Federal Reserve Bank of Minneapolis, pp. 169-210.

[4] Burdett, Kenneth, Alberto Trejos, and Randall Wright (2001). "Cigarette Money," Journal of Economic Theory 99, 117-142.

[5] Einzig, Paul (1966). Primitive Money and its Ethnological, Historical and Economic Aspects. Pergamon Press, Second Edition.

[6] Friedman, Milton (1960). A Program for Monetary Stability. Fordham University Press.

[7] Kiyotaki, Nobuhiro, and Randall Wright (1989). "On money as a medium of exchange," Journal of Political Economy 97, 927-954.

[8] Kiyotaki, Nobuhiro, and Randall Wright (1993). "A search-theoretic approach to monetary economics," American Economic Review 83, 63-77.

[9] Lagos, Ricardo and Randall Wright (2000). "A Unified Framework for Monetary Theory and Policy Analysis," mimeo, New York University and University of Pennsylvania.

[10] Sargent, Thomas J., and Neil Wallace (1983). "A Model of Commodity Money," Journal of Monetary Economics 12, 163-187.

[11] Shi, Shouyong (1999). "Search, Inflation and Capital Accumulation," Journal of Monetary Economics 44, 81-103.

[12] Wallace, Neil (1980). "The overlapping generations model of fiat money," in Models of Monetary Economies, John Kareken and Neil Wallace, eds., Federal Reserve Bank of Minneapolis, pp. 49-82. 